\title{
Gross cystic disease fluid protein 15 (GCDFP-15) expression in breast cancer subtypes
}

\author{
Silvia Darb-Esfahani ${ }^{1 *}$, Gunter von Minckwitz ${ }^{2,3}$, Carsten Denkert ${ }^{1}$, Beyhan Ataseven ${ }^{4}$, Bernhard Högel ${ }^{5}$, \\ Keyur Mehta ${ }^{2}$, Gabriele Kaltenecker ${ }^{6}$, Thomas Rüdiger ${ }^{7}$, Berit Pfitzner ${ }^{1}$, Kornelia Kittel ${ }^{8}$, Bettina Fiedler ${ }^{9}$, \\ Klaus Baumann ${ }^{10}$, Roland Moll ${ }^{11}$, Manfred Dietel ${ }^{1}$, Holger Eidtmann ${ }^{12}$, Christoph Thomssen ${ }^{13}$ and Sibylle Loibl ${ }^{4}$
}

\begin{abstract}
Background: Gross cystic disease fluid protein 15 (GCDFP-15), which is regulated by the androgen receptor (AR), is a diagnostic marker for mammary differentiation in histopathology. We determined the expression of GCDFP-15 in breast cancer subtypes, its potential prognostic and predictive value, as well as its relationship to AR expression.

Methods: 602 pre-therapeutic breast cancer core biopsies from the phase III randomized neoadjuvant GeparTrio trial (NCT00544765) were investigated for GCDFP-15 expression by immunohistochemistry. Expression data were correlated with disease-free (DFS) and overall survival (OS) time as well as pathological complete response (pCR) to neoadjuvant chemotherapy.
\end{abstract}

Results: 239 tumors (39.7\%) were GCDFP-15 positive. GCDFP-15 expression was positively linked to hormone receptor $(\mathrm{HR})$ and HER2 positive tumor type, while most triple negative carcinomas were negative $(p<0.0001)$. GCDFP-15 was

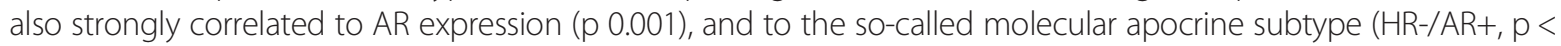
0.0001). Higher rates of GCDFP-15 positivity were seen in tumors of lower grade $(<0.0001)$ and negative nodal status ( $p=0.008)$. GCDFP-15 positive tumors tended to have a more favourable prognosis than GCDFP-15 negative tumors (DFS $(p=0.052)$ and OS $(p=0.044)$ ), which was not independent from other factors in multivariate analysis. GCDFP-15 expression was not linked to pCR. Histological apocrine differentiation was frequent in molecular apocrine carcinomas (60.7\%), and was associated with GCDFP-15 within this group ( $p=0.039)$.

Conclusions: GCDFP-15 expression is higher in tumors with favorable prognostic features. GCDFP-15 expression is further a frequent feature of AR positive tumors and the molecular apocrine subtype. It might have reduced sensitivity as a diagnostic marker for mammary differentiation in triple negative tumors as compared to HR or HER2 positive tumor types.

Keywords: GCDFP-15, Breast cancer, Neoadjuvant chemotherapy, Apocrine, CUP

\section{Background}

Gross cystic disease fluid protein 15 (GCDFP-15, syn. prolactin-inducible protein, PIP) is a $15 \mathrm{kDa}$ protein that was originally detected in the cystic fluid from cystic mastopathy [1]. It is not expressed in normal ductal or lobular epithelium but in apocrine metaplasia of the breast [2]. Apart from breast cancer, only very few tumors, such as prostate cancer and carcinomas of the skin appendages express GCDFP-15 [3]. It is therefore

\footnotetext{
* Correspondence: silvia.darb-esfahani@charite.de

${ }^{1}$ Institute of Pathology, Charité Universitätsmedizin Berlin, Charitéplatz 1, 10117 Berlin, Germany

Full list of author information is available at the end of the article
}

highly specific for mammary differentiation in females, and is frequently used as an immunohistochemical marker for the evaluation of a potential mammary origin of metastatic carcinoma of unknown primary site. The expression of GCDFP-15 is regulated by the androgen receptor (AR) [4], however, little is known about its function. A recent study on gene expression profiles in androgen-stimulated, GDCFP-15 expressing versus GCDFP-15 non-expressing breast cancer cell lines, reported an up-regulation of proapoptotic and anti-proliferative genes along with GCDFP15 [5]. In carcinomas of the breast, GCDFP-15 is also used as a marker for apocrine differentiation [2,6-9]. Apocrine breast carcinoma is a rare subtype of invasive ductal 
carcinoma, which is primarily defined by morphological features such as abundant eosinophilic and granular cytoplasm, and shows frequent expression of the androgen receptor (AR) [10]. Some years ago a so-called molecular apocrine subset of breast carcinoma has been defined by gene expression analysis, and was characterized by active $\mathrm{AR}$ and weak or absent estrogen receptor (ER) signalling [11]. In this study, all tumors that were assigned to the molecular apocrine group had strong morphological features of apocrine differentiation. The existence of the molecular apocrine subtype has since then been reproduced $[12,13]$. However, its clinical impact is conflictive to date.

We used a large and well-characterized cohort of breast cancer patients who underwent anthracycline/taxane-based neoadjuvant chemotherapy (NACT) in the phase III randomized GeparTrio trial (NCT00544765) to investigate the distribution of GCDFP-15 expression in biological subtypes of breast cancer, its potential prognostic and predictive value, as well as its relationship to AR expression. GCDFP-15 expression and biological tumor types were determined by immunohistochemistry in pre-therapeutic breast cancer core biopsies.

\section{Methods}

\section{Study Population}

Samples from the prospective neoadjuvant phase III GeparTrio study (NCT00544765) and the GeparTrio pilot study performed by the German Breast Group (GBG), Neu-Isenburg, Germany were used. Patients were treated with anthracycline/taxane-based NACT. The details of study setup and treatments have been published before [14-17]. HER2 positive patients had not received trastuzumab in GeparTrio as this was not the standard of care during the study period. Baseline clinico-pathological data as well as data on hormone receptor (HR) status were extracted from the study databases. Centrally evaluated data on HER2 expression (based on immunohistochemistry and silver-enhanced in situ hybridization according to ASCO/CAP guidelines [18] were used, as HER2 determination was not yet fully established in all pathologic laboratories at the time the study was conducted. Grading and histology were also centrally determined; local data on HR expression were used and substituted with central data if missing (central evaluation: Institute of Pathology, Charité Berlin). Consistent with the current practise when the trial was performed, HR positivity was defined as estrogen (ER) or progesterone receptor (PR) expression in more than $10 \%$ of tumor cells. We also exploratorily applied the cutoff of $1 \%$ currently recommended by ASCO/CAP [19]; use of this definition of HR positivity yielded quite similar results (see results section). Data on AR expression had been obtained from 545 cases in a previous study [20]. In brief, AR staining intensity as well as percentage of stained cells was multiplied to an immunoreactivity score (IRS), ranging from 0 (negative in all cells) to 12 (strongly expressed in more than $80 \%$ of cells). Cases with an IRS from 0-3 were scored as AR negative, opposed to cases with an IRS of 4-12 (=AR positive). Definition of pathological complete response (pCR) was complete absence of invasive tumour cells in the breast and lymph nodes as assessed at the time of surgery by the local pathologist (ypT0/Tis; ypN0). Data on disease-free survival (DSF) and overall survival (OS) were available for 570 patients, with mean DFS of 3.08 years and mean OS of 3.42 years. The baseline demographic and clinical characteristics of the patients with tissue available for this translational research project are shown in Table 1 . The protocol was reviewed and approved by all responsible ethics committees (Additional file 1: Table S1). All patients provided written informed consent for anonymized subsequent translational research.

\section{Immunohistochemistry}

Construction of a tissue micro array (TMA) of pretherapeutic core biopsies has been explained previously [20]. Immunohistochemistry was performed on a Ventana BenchMark XT instrument (Ventana Medical Systems Inc., Tucson, AZ, USA) after pre-treatment with protease using a mouse monoclonal antibody directed against human GCDFP-15 (clone D6, dilution 1:400, Covance, Princeton, NJ, USA). For visualization, the iView DAB detection kit (Ventana Medical Systems Inc.) was used. Stained slides were digitized and evaluated on the computer monitor with support of the TMA Evaluator software (VMScope GmbH, Berlin, Germany) by a board certified pathologist (S. D.-E.). Both staining intensity and the percentage of stained tumor cells were evaluated and combined to an IRS (see previous chapter).

\section{Statistical evaluation}

Statistical analysis was performed using SPSS Statistics 19 (IBM Corporation, Somers, NY, USA). In logistic regression analyses, significance of the correlation with pCR was assessed by the Wald test. Survival analyses were performed with the Kaplan-Meier method and univariate logrank test and with Cox regression analysis for multivariate tests. The association between GCDFP-15 expression and clinico-pathological factors, biological tumor types, and AR expression was analysed by Fisher's exact test or Pearson's chi square test, as indicated. All tests were two-sided, and $\mathrm{p}$-values $<0.05$ were considered as significant.

\section{Results}

\section{GCDFP-15 expression pattern in human breast carcinomas}

844 TMA spots were evaluated (one for each individual tumor), whereas 203 spots contained no tumor cells (24.1\%), and 39 spots contained no tissue (4.6\%), resulting in 602 informative cases (Figure 1). Consistent with 
Table 1 Association of GCDFP-15 expression with baseline clinico-pathological parameters

\begin{tabular}{|c|c|c|c|c|}
\hline & Total $(100 \%)$ & GCDFP-15 negative & GCDFP-15 positive & $\mathrm{p}$ \\
\hline Total & 602 & $363(60.3 \%)$ & 239 (39.7\%) & - \\
\hline HR $(n=576)$ & & & & $0.034^{\mathrm{a}}$ \\
\hline Negative & 175 & $116(66.3 \%)$ & $59(33.7 \%)$ & \\
\hline Positive & 401 & $228(56.9 \%)$ & $173(43.1 \%)$ & \\
\hline HER2 $(n=576)$ & & & & $0.035^{\mathrm{a}}$ \\
\hline Negative & 478 & $299(62.6 \%)$ & $179(37.4 \%)$ & \\
\hline Positive & 116 & $60(51.7 \%)$ & $56(48.3 \%)$ & \\
\hline Biological tumor types $(n=569)$ & & & & $0.001^{b}$ \\
\hline $\mathrm{HR}+/ \mathrm{HER} 2-$ & 328 & $189(57.6 \%)$ & $139(42.4 \%)$ & \\
\hline $\mathrm{HR}+/ \mathrm{HER}+$ & 68 & $37(54.4 \%)$ & $31(45.6 \%)$ & \\
\hline HR-/HER2+ & 43 & $19(44.2 \%)$ & $24(55.8 \%)$ & \\
\hline HR-/HER2- & 130 & $96(73.8 \%)$ & $34(26.2 \%)$ & \\
\hline $\mathbf{A R}(n=545)$ & & & & $<0.0001^{a}$ \\
\hline Negative & 238 & $180(75.6 \%)$ & $58(24.4 \%)$ & \\
\hline Positive & 307 & $149(48.5 \%)$ & $158(51.5 \%)$ & \\
\hline Age $(n=602)$ & & & & $0.066^{\mathrm{a}}$ \\
\hline$<50$ years & 273 & $176(64.5 \%)$ & 97 (35.5\%) & \\
\hline$>=50$ years & 329 & $187(56.8 \%)$ & $142(43.2 \%)$ & \\
\hline Histological type $(n=602)$ & & & & $0.090^{\mathrm{b}}$ \\
\hline Ductal/others & 554 & $340(61.4 \%)$ & $214(38.6 \%)$ & \\
\hline Lobular & 48 & $23(47.9 \%)$ & $25(52.1 \%)$ & \\
\hline Grading $(n=601)$ & & & & $<0.0001^{\mathrm{a}}$ \\
\hline G1-2 & 463 & $261(56.4 \%)$ & $202(43.6 \%)$ & \\
\hline G3 & 138 & $102(73.9 \%)$ & $36(26.1 \%)$ & \\
\hline cT $(n=591)$ & & & & $0.181^{a}$ \\
\hline $\mathrm{cT} 1-2$ & 395 & $230(58.2 \%)$ & $165(41.8 \%)$ & \\
\hline cT3-4 & 196 & $126(64.3 \%)$ & $70(35.7 \%)$ & \\
\hline $\mathbf{c N}(n=583)$ & & & & $0.008^{a}$ \\
\hline $\mathrm{cNO}$ & 261 & $141(54.0 \%)$ & $120(46.0 \%)$ & \\
\hline $\mathrm{cN}+$ & 322 & 209 (64.9\%) & $113(35.1 \%)$ & \\
\hline
\end{tabular}

${ }^{\mathrm{a}}$ Fisher's exact test.

bearson's chi square test.

previous reports [21,22], we found GCDPF-15 restricted to the cytoplasm of tumor cells. Positive tumors mostly displayed a weak to moderate stain in variable fractions of cells, and a patchy or mosaic-like pattern could be frequently found (Figure 2A, B). Sparse tumors showed diffuse staining (Figure 2C). In the majority of samples however, GCDFP-15 expression was totally absent $(n=363(60.3 \%$, Figure $2 D))$. We therefore decided to score each apparent staining as positive and opposed it to totally lacking staining. No GCDFP-15 staining was seen in non-epithelial cells such as stromal or inflammatory cells.

\section{Association with biological tumor types and clinico- pathological factors}

GCDFP-15 expression was significantly enriched in tumors with certain biological characteristics. There was a modest increase of GDCFP-15 expression in HR and in HER2 positive tumors (Table 1). GCDFP-15 positivity rate was $43.1 \%$ in HR positive tumors and $33.7 \%$ in HR negative tumors $(\mathrm{p}=0.034)$, and $48.3 \%$ HER2 positive carcinomas expressed GCDFP-15 as opposed to 37.4\% HER2 negative tumors $(\mathrm{p}=0.035)$. Consequently, GCDFP-15 was also differentially distributed among biological tumor types, as defined by HR and HER2 status: frequency of 


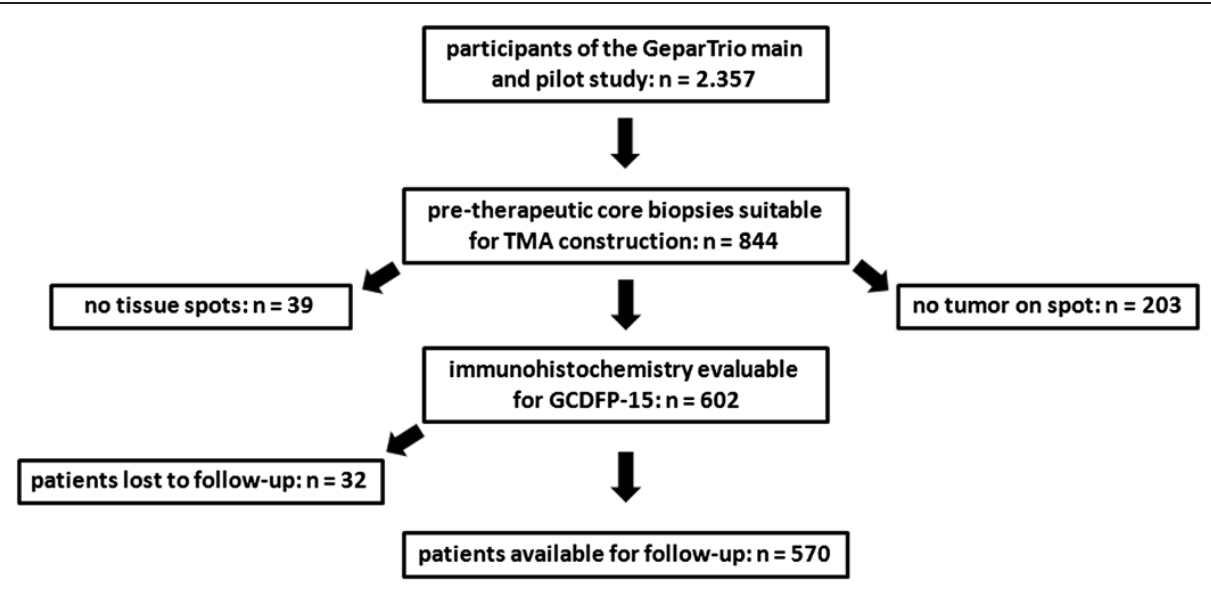

Figure 1 Consort diagram.

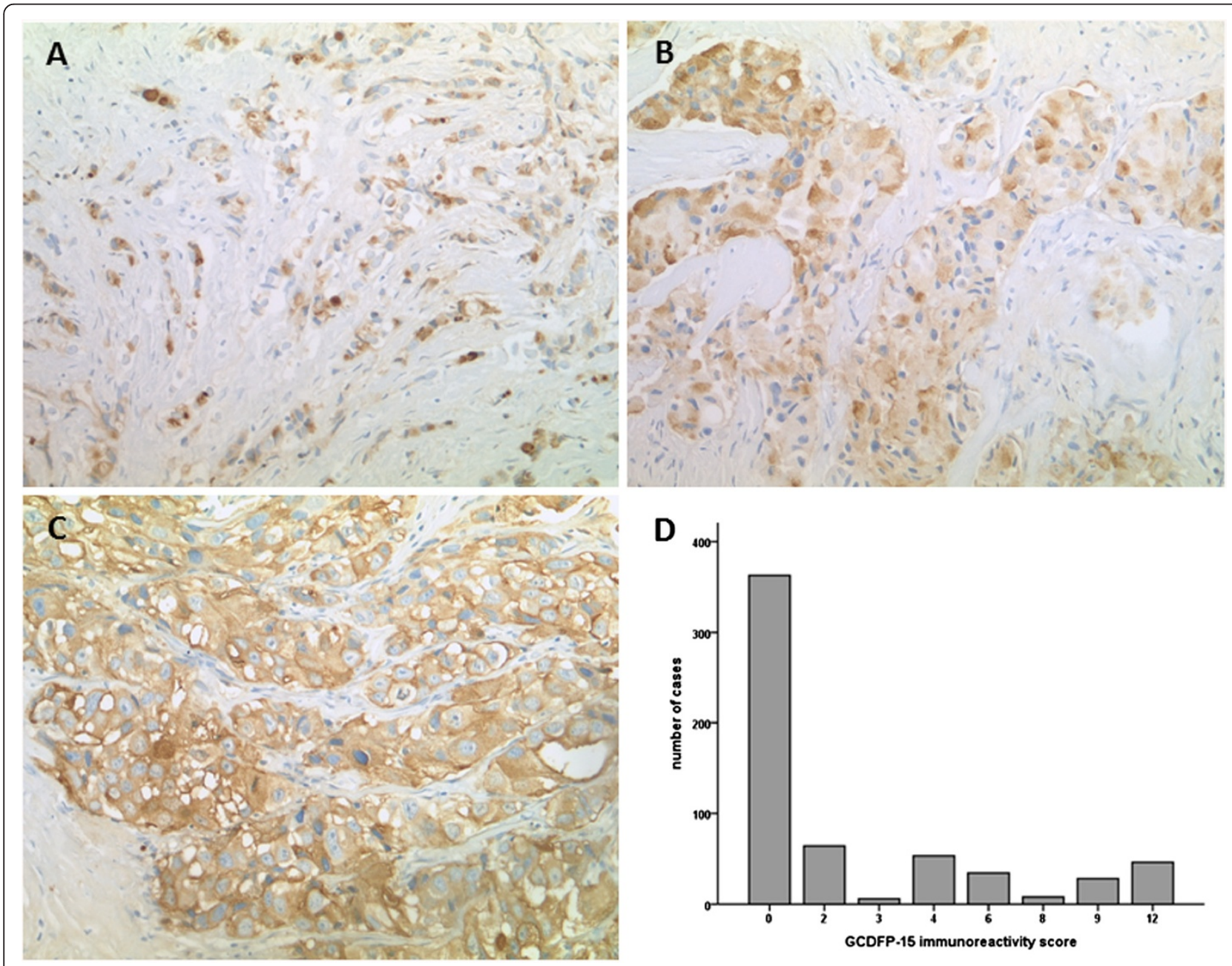

Figure 2 Immunohistochemical expression pattern of GCDFP-15 in breast cancer. A Tumor cells of an invasive lobular carcinoma, arranged in indian file pattern and exhibiting moderate cytoplasmic staining for GDCFP-15 B Solid carcinoma nests with patchy, mosaic-like pattern of GCDFP-15 expression C Diffuse GCDFP-15 expression in a poorly differentiated ductal carcinoma D Distribution of GCDFP-15 immunoreactivity scores (IRS) in the study group. The majority of cases did not display any staining (IRS $=0$ ); in the remaining carcinomas, IRS values were equally distributed; the cut-off was set between IRS $=0$ and IRS $=2$. 
positive tumors was significantly higher in luminal subtypes (HR+/HER2-: 42.4\%, HR+/HER2: 45.6\%) as well in HER2 positive tumors (HR-/HER2+: $55.8 \%$ ) as opposed to in triple negative breast carcinomas (TNBC, HR-/HER2-: $26.2 \%, \mathrm{p}=0.001$, Table 1). Consistent with GCDFP-15 being a downstream target gene of AR [4], there was a strong association between GCDFP-15 and AR expression. $51.5 \%$ of AR positive tumors were also positive for GCDFP-15, whereas only $24 \%$ of AR negative carcinomas showed GCDFP-15 staining ( $\mathrm{p}<0.0001)$. As AR is a frequent feature of apocrine tumor differentiation and GCDFP-15 has also been proposed as a marker for apocrine differentiation, we tested GCDFP-15 expression for an association with the so-called molecular apocrine subtype (HR-/AR+), as described by Farmer et al. [11]. Tumor types according to Farmer were grouped as follows: $\mathrm{HR}+(\mathrm{AR}+/-), \mathrm{n}=365), \mathrm{HR}-/ \mathrm{AR}+(\mathrm{n}=56)$, and HR-/AR- $(\mathrm{n}=101)$. HER2 positivity was more frequent in molecular apocrine carcinomas $(42.9 \%$ as opposed to $17.1 \%$ in $\mathrm{HR}+(\mathrm{AR}+/-)$ and $15.8 \%$ in HR-/AR-, $\mathrm{p}<$ $0.0001)$. There was a significant association between GCDFP-15 and molecular apocrine subtype, with 67.9\% GCDFP-15 positive cases in this group $(\mathrm{p}<0.0001)$. $41.6 \%$ of $\mathrm{HR}+(\mathrm{AR}+/-)$ tumors were also positive for GCDFP-15, while the rate of GCDFP-15 positive cases in the subgroup that was completely negative for steroid hormone receptors (HR-/AR-) with $18.8 \%$ was lower than the one in triple negative tumors (HR-/HER2-, $26.2 \%$, Table 1). GCDFP-15 was further associated with certain favorable tumor features, such as lower tumor grade $(\mathrm{p}<0.0001)$, and negative nodal status $(\mathrm{p}=0.008$, Table 1). Using the currently by ASCO/CAP guidelines [19] recommended cutoff for ER/PR evaluation $(<1 \%$ stained tumor cells $=$ negative, $>=1 \%$ stained tumor cells $=$ positive) we obtained similar results: HR positivity rate in the total study group was 79.5\%, GCDFP-15 expression still was associated with HR positivity $(\mathrm{p}=0.046)$ and with molecular apocrine tumor type $(\mathrm{p}<0.0001)$, although the rate of molecular apocrine tumors was now decreased to $6.5 \%$.

\section{Morphological features of molecular apocrine carcinomas}

We further wondered whether the molecular apocrine subtype was showed a distinct morphology and re-evaluated hematoxylin/eosin-stained large sections of pre-therapeutic core biopsies according to apocrine differentiation. Criteria were based on Vranic et al. (2013) [6] and included tumors with large nuclei and characteristic abundant eosinophilic granular or foamy cytoplasm (type A, type B cells). Indeed, molecular apocrine carcinomas were quite frequently of apocrine phenotype (34/56, 60.7\%, Figure 3A). Two carcinomas of pleomorphic lobular subtype, a poorly differentiated subgroup of lobular-invasive carcinoma reported to cluster with molecular apocrine tumors by gene expression analysis [23], were among them (Figure 3B). Histologically apocrine carcinomas with $\mathrm{HR}-/ \mathrm{AR}+$ profile were GCDFP-15 positive in most cases $(27 / 34,71.1 \%, \mathrm{p}=$ 0.039; Figure 3A, B).

\section{Prognostic impact of GCDFP-15 expression}

GCDFP-15 expression was also studied for a potential prognostic impact. GCDFP-15 positive tumors tended to have a more favourable prognosis than GCDFP-15 negative tumors (DFS $(\mathrm{p}=0.052)$ and $\mathrm{OS}(\mathrm{p}=0.044))$ in the study group (Figure 4A, B, Table 2). Explorative multivariate Cox regression analysis including HR and HER2 expression, age, nodal stage, and grading showed that GCDFP-15 expression was not an independent prognostic factor for OS $(\mathrm{HR}=0.67,95 \% \mathrm{CI}=0.37-1.20, \mathrm{p}=$ 0.179 , not shown). Similarly, GCDFP-15 was not a significant prognostic marker for OS or DFS within the biological tumor types (HR+/HER-, HR+/HER2+, HR-/ HER2+, HR-/HER2-) or in Farmer tumor types (HR + $(\mathrm{AR}+/-), \mathrm{HR}-/ \mathrm{AR}+, \mathrm{HR}-/ \mathrm{AR}-; \mathrm{p}<0.05$ for each test, not shown). Farmer tumor types by themselves were also prognostic for DSF and OS (log rank $\mathrm{p}=0.024$ for each), however a survival difference was seen only between HR- and HR + tumors, and was irrespective of an additional AR expression (data not shown). The following established prognostic makers for DFS and/or OS were significant in univariate analysis in the GeparTrio cohort as well: HR expression, biological tumor types, age, tumor grade, $\mathrm{cT}$, and $\mathrm{cN}$ (Table 2).

\section{Predictive value of GCDFP-15 expression}

We further evaluated if GCDFP-15 expression might have predictive value for response to NACT and performed logistic regression analysis. In the total study group, there was a non-significant trend towards a reduced probability of $\mathrm{pCR}$ in GCDFP- 15 positive tumors (pCR rate $21.2 \%$ vs $15.9 \%, \mathrm{OR}=0.70,95 \% \mathrm{CI}=0.46-1.08, \mathrm{p}=0.106$, Table 3 ). GCDFP15 expression was not indicative of response to NACT within biological tumor types or Farmer tumor types ( $<0.05$ for each test, not shown). Farmer tumor types were significantly predictive for $\mathrm{pCR}$, however similarly to the survival analysis, only the HR status was relevant for the predictive effect, and there was no difference between molecular apocrine (HR-/AR+) and HR-/AR- tumors: odds ratio (OR) of HR-/AR + 4.1 (95\% CI 2.1-7.7), pCR rate 33.9\%; OR of HR-/AR- 4.2 (95\% CI 2.5-7.1), pCR rate $34.7 \%$, as compared to $H R+$, respectively $(\mathrm{p}<0.0001$, pCR rate $11.2 \%)$. Already known predictive factors were also significant in our cohort: age, histological type, grade, HR expression, HER2 expression, and biological tumor types (Table 3).

\section{Discussion}

We investigated the expression of GCDFP-15 in a large and well-characterized clinical trial cohort of breast 


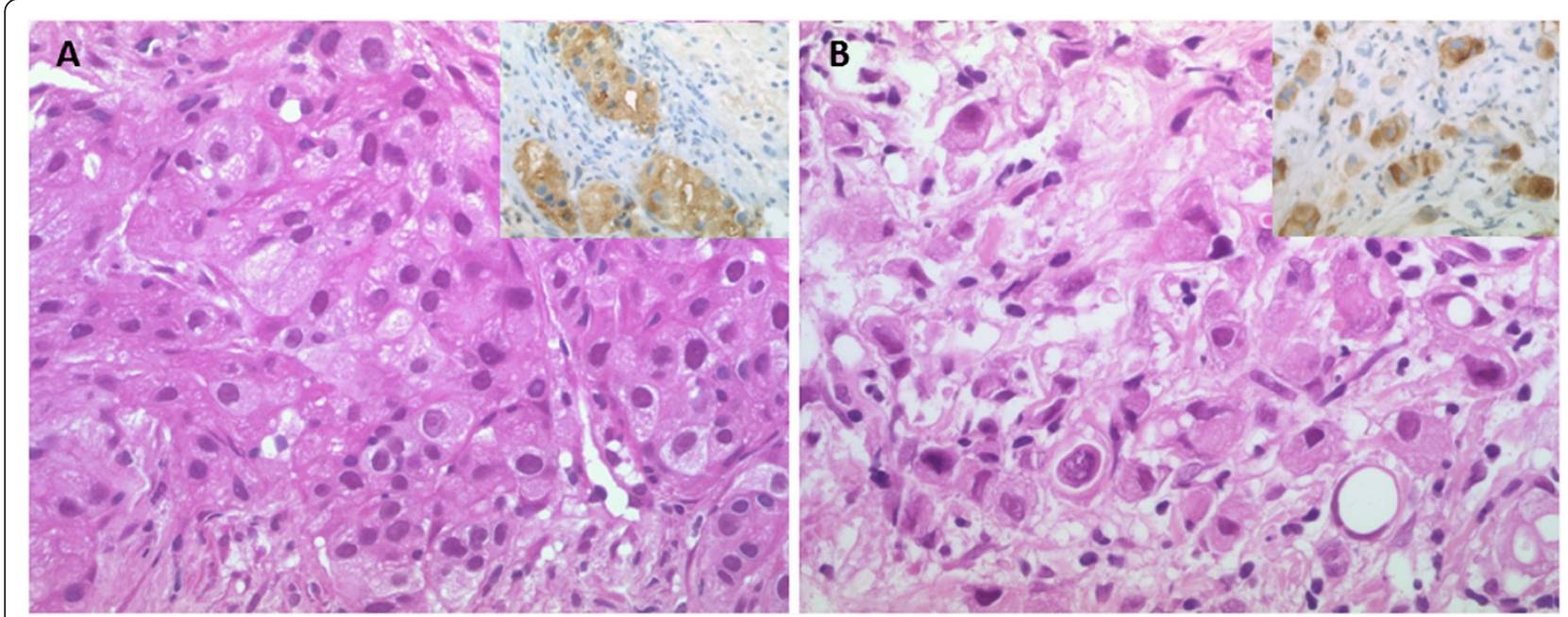

Figure 3 Morphology of molecular apocrine carcinomas. A Apocrine carcinoma with abundant eosinophilic granular cytoplasm exhibiting diffuse GCDFP-15 expression (insert) B Pleomorphic lobular carcinoma with dyscohesive growth of large cells with highly atypical nuclei and eosinophilic granular cytoplasm, strong diffuse GCDFP-15 expression is seen by immunohistochemistry (insert).

carcinomas treated with NACT with a special emphasis on its distribution in breast cancer subtypes and its prognostic impact. We found that GCDFP-15 was increased in HR positive as well as in HER2 positive subtypes as compared to TNBC (HR-/HER2-). GCDFP-15 expression was not predictive for response to NACT. Although GCDFP-15 was a favorable prognostic factor for DFS and OS in univariate analysis, this impact was not independent from other factors and not evident within breast cancers subtypes. GCDFP-15 was furthermore strongly associated with AR and therefore enriched in the so-called molecular apocrine breast cancer subtype.

Although widely used as a diagnostic marker for breast carcinoma in pathology, the prognostic value of GCDFP15 has not been systematically evaluated to date. Pagani (1994), in a small case series of 33 breast cancers found evidence of a longer relapse-free survival in patients with tumors positive for GCDFP15 gene expression [24]. Fritzsche et al. (2007) reported GCDFP-15 as a positive prognostic factor in a cohort of 165 carcinomas [25].
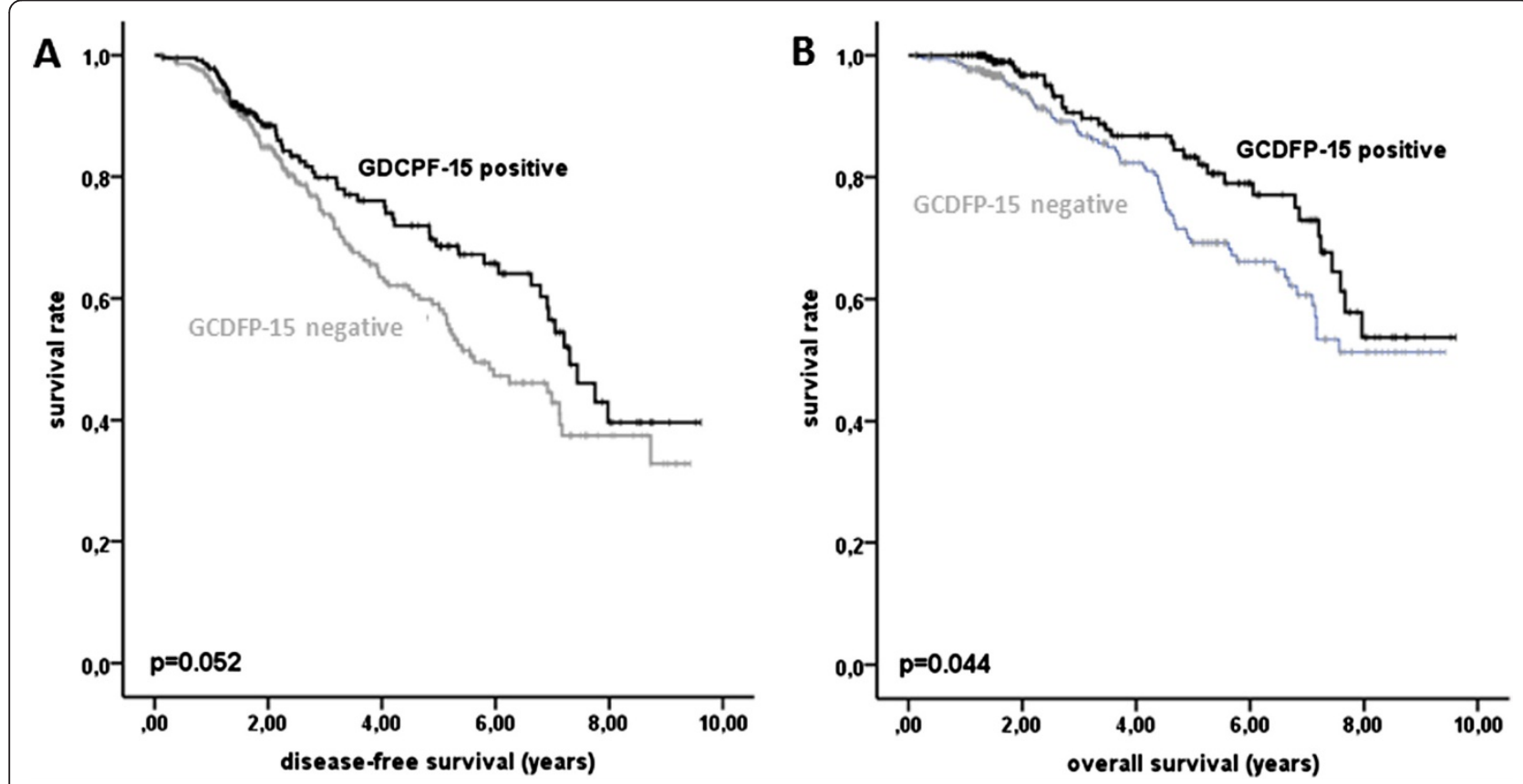

Figure 4 Survival analysis A, B DFS and OS in dependence of GCDFP-15 expression in the study group. 
Table 2 Univariate survival analysis

\begin{tabular}{|c|c|c|c|c|c|c|}
\hline & & DFS & & & OS & \\
\hline & $\%$ events & Mean survival, years (SE) & $p$ & $\%$ events & Mean survival, years (SE) & $p$ \\
\hline GCDFP-15 & & & & & & 0.044 \\
\hline Negative & 30.3 & $5.85(0.24)$ & & 19.3 & $7.07(0.23)$ & \\
\hline Positive & 25.1 & $6.67(0.30)$ & 0.052 & 13.9 & $7.75(0.27)$ & \\
\hline$H R$ & & & & & & 0.013 \\
\hline Negative & 35.6 & $5.53(0.36)$ & & 23.3 & $6.75(0.34)$ & \\
\hline Positive & 25.0 & $6.48(0.23)$ & 0.004 & 19.3 & $7.67(0.34)$ & \\
\hline HER2 & & & & & & 0.686 \\
\hline Negative & 26.3 & $6.31(0.22)$ & & 16.0 & $7.46(0.21)$ & \\
\hline Positive & 37.0 & $5.64(0.40)$ & 0.114 & 22.3 & $7.06(0.24)$ & \\
\hline Biological tumor types & & & & & & 0.084 \\
\hline $\mathrm{HR}+/ \mathrm{HER} 2-$ & 23.2 & $6.61(0.25)$ & & 13.7 & $7.71(0.24)$ & \\
\hline HR+/HER2+ & 33.8 & $5.86(0.52)$ & & 16.9 & $7.45(0.43)$ & \\
\hline HR-/HER2+ & 43.6 & $4.98(0.60)$ & & 30.8 & $6.23(0.53)$ & \\
\hline HR-/HER2- & 33.3 & $5.59(0.42)$ & 0.011 & 21.1 & $6.86(0.41)$ & \\
\hline Age & & & & & & 0.046 \\
\hline$<50$ years & 24.9 & $6.48(0.28)$ & & 12.8 & $7.79(0.25)$ & \\
\hline$>=50$ years & 31.0 & $5.93(0.26)$ & 0.120 & 20.8 & $7.06(0.24)$ & \\
\hline Histological type & & & & & & 0.915 \\
\hline Ductal/others & 28.8 & $6.15(0.20)$ & & 17.2 & $7.40(0.19)$ & \\
\hline Lobular & 10.0 & $6.42(0.55)$ & 0.309 & 17.4 & $6.82(0.55)$ & \\
\hline grading & & & & & & 0.043 \\
\hline G1-2 & 26.4 & $6.42(0.22)$ & & 16.1 & $7.54(0.20)$ & \\
\hline G3 & 34.6 & $5.35(0.41)$ & 0.005 & 20.8 & $6.70(0.40)$ & \\
\hline cT & & & & & & 0.001 \\
\hline $\mathrm{cT} 1-2$ & 20.8 & $6.90(0.24)$ & & 12.4 & $7.88(0.22)$ & \\
\hline cT3-4 & 43.9 & $4.93(0.31)$ & $<0.0001$ & 26.7 & $6.65(0.31)$ & \\
\hline $\mathrm{cN}$ & & & & & & $<0.0001$ \\
\hline $\mathrm{cNO}$ & 22.4 & $6.91(0.28)$ & & 11.4 & $8.12(0.24)$ & \\
\hline $\mathrm{cN}+$ & 33.9 & $5.56(0.26)$ & $<0.0001$ & 22.5 & $6.75(0.25)$ & \\
\hline
\end{tabular}

DFS: disease-free survival.

OS: overall survival.

SE: standard error.

However, in this study the prognostic impact of GCDFP15 expression was not investigated for each biological tumor type separately. Due to the very different molecular biology of those breast cancer subtypes, biomarkers may have quite varying prognostic implication within the subtypes. We show here that the prognostic impact of GCDFP-15 is most likely a bystander effect of its association with other factors, such as HR expression, nodal stage, and tumor grade. Our study thereby confirms previous findings of an association between GCDFP-15 expression and features of good-prognosis tumors [7,22,26], and it might be speculated that GCDFP-15 parallels the expression of its regulatory factor $\mathrm{AR}$, which is also linked to favorable prognostic clinico-pathological features, as we showed previously [20]. We further found that GCDFP-15 is differentially expressed in breast cancer subtypes and is enriched in luminal and HER2 positive carcinomas, while being relatively sparse in TNBC. Similarly, Huo et al. (2013) reported a rather low percentage of GCDFP-15 positives in primary (14\%) and metastatic TNBC (21\%) [19]. Lewis et al. (2011) found even higher rates of GCDFP-15 expression than us in luminal (65-71\%) and in HER2 positive carcinomas (64\%), and found only one out 33 TNBC (basal-like and unclassified triple negative tumors) to be positive for GCDFP-15, however, their cohort being relatively small, might have underestimated the frequency of 
Table 3 Univariate logistic regression: association with pCR

\begin{tabular}{|c|c|c|c|c|c|c|}
\hline & $\mathbf{n}$ & Events & $\% \mathrm{pCR}$ & OR & $95 \% \mathrm{Cl}$ & $p$ \\
\hline GCDFP-15 & & & & & & 0.106 \\
\hline Negative & 363 & 77 & 21.2 & 1 & - & \\
\hline Positive & 239 & 38 & 15.9 & 0.70 & $0.46-1.08$ & \\
\hline HR & & & & & & $<0.0001$ \\
\hline Negative & 175 & 61 & 34.9 & 1 & - & \\
\hline Positive & 401 & 45 & 11.2 & 0.24 & $0.15-0.37$ & \\
\hline HER2 & & & & & & $<0.0001$ \\
\hline Negative & 478 & 77 & 16.1 & 1 & - & \\
\hline Positive & 116 & 36 & 31.0 & 2.34 & $1.48-3.72$ & \\
\hline Biological tumor types & & & & & & $<0.0001$ \\
\hline $\mathrm{HR}+/ \mathrm{HER} 2-$ & 328 & 28 & 8.5 & 1 & - & \\
\hline HER2+ $(\mathrm{HR}+/-)$ & 111 & 34 & 30.6 & 4.73 & $2.70-8.28$ & \\
\hline HR-/HER2- & 130 & 43 & 33.1 & 5.30 & $3.11-0.02$ & \\
\hline Age & & & & & & 0.001 \\
\hline$<50$ years & 273 & 68 & 24.9 & 1 & - & \\
\hline$>=50$ years & 329 & 47 & 14.3 & 0.50 & $0.33-0.76$ & \\
\hline \multicolumn{7}{|l|}{ Histological type } \\
\hline Ductal/others & 554 & 113 & 20.4 & 1 & - & \\
\hline Lobular & 48 & 2 & 4.2 & 0.17 & $0.04-0.71$ & \\
\hline Grading & & & & & & $<0.0001$ \\
\hline G1-2 & 463 & 74 & 16.0 & 1 & - & \\
\hline G3 & 138 & 41 & 29.7 & 2.22 & $1.43-3.46$ & \\
\hline сT & & & & & & 0.098 \\
\hline cT1-2 & 395 & 83 & 21.0 & 1 & - & \\
\hline CT3-4 & 196 & 30 & 15.3 & 0.68 & $0.43-1.07$ & \\
\hline $\mathrm{cN}$ & & & & & & 0.809 \\
\hline $\mathrm{cNO}$ & 261 & 49 & 18.8 & 1 & - & \\
\hline $\mathrm{cN}+$ & 322 & 63 & 19.6 & 1.05 & $0.70-1.59$ & \\
\hline
\end{tabular}

OR: Odd's ratio.

$\mathrm{Cl}$ : confidence interval.

GCDFP-15 positivity in TNBC [22]. Taken together, these data warrant care if GCDFP-15 is used as a diagnostic marker for mammary differentiation of metastases of a cancer of unknown primary (CUP) because a significant proportion of breast cancers, particularly TNBC might be negative. An extended panel of immunohistochemical markers for mammary differentiation should be used to increase sensitivity. We show an enrichment of GCDFP15 expression in HER2 positive tumors and a strong association with $\mathrm{AR}$ expression, and are therefore in line with previous reports [7,22,27]. Not surprisingly, we further found GCDFP-15 to be elevated in the so-called molecular apocrine carcinomas that are defined by AR expression in the absence of HR expression [11]. In our study histological apocrine differentiation was found in $60.7 \%$ of molecular apocrine carcinomas; additionally, GCDFP-15 within the molecular apocrine subgroup was associated with histological signs of apocrine differentiation, which suggests that ER/PR, AR, and GCDFP-15 expression are helpful markers to confirm apocrine differentiation in morphologically conspicuous cases. On the other hand, 39.3\% of HR-/AR + carcinomas did not show apocrine morphology in our cohort, which indicates that molecularly and morphologically defined apocrine groups overlap only partly. The clinical significance of the molecular apocrine subtype is not clear to date and remains to be determined as proposed by the current WHO Classification of Tumors of the Breast [28] (2012), similarly conflictive data exist regarding the prognostic impact of histologically defined apocrine subtype (reviewed by Vranic et al. [6]). Our study does not point to a particular prognosis or therapy response of HR-/AR + carcinomas, as $\mathrm{pCR}$ rate and survival 
times were quite similar to those in HR- tumors without AR expression. Interestingly, HER2 expression seems to interact with $A R$ in $H R$ negative tumors in prognostic terms, as in our previous study in the same cohort, AR positivity was a positive prognostic factor only in the molecular subgroup of triple negative breast cancer (as defined by ER/PR/HER2 negativity).

A limitation of our study is the reduced sample size in the HER2 positive tumor types (HR+/HER2+: $n=68$, HR-/HER2+: $n=43$ ), which might hamper the detection of a differential expression of GCDFP-15 or a prognostic impact of GCDFP-15 expression within those tumor types. Furthermore, relatively short follow-up times indicate that survival analysis should be interpreted cautiously (the GeparTrio study not being powered for survival as a primary end point). An additional limitation might be that we used a TMA constructed out of core biopsies, which in some cases contained only few tumor cells and which together with the focal expression pattern of GCDFP-15 might result in a reduced sensitivity for detection of GCDFP-15 expression in some tumors. However, the rate of GCDFP-15 expression in our study group (39.7\%) was in the range reported in the literature [21,26]. Only 602 out of 2.357 patients in the original GeparTrio studies could be included for this project; however this is still the largest study on GCDFP-15 expression to date.

\section{Conclusion}

GCDFP-15 is expressed in all major biological breast cancer subtypes, and may be particularly useful as a diagnostic marker for mammary differentiation in HR and HER2 positive tumors, while there is reduced sensitivity in the triple negative subset. Due to its strong link to AR expression it may also be a marker for the so-called molecular apocrine subtype. GCDFP-15 is linked to clinico-pathological factors that indicate a better patient outcome, but is by itself no independent prognostic factor and is not predictive of response to anthracycline/taxane-based NACT.

\section{Additional file}

Additional file 1: Ethics committees that approved the GeparTrio study.

\section{Competing interests}

The authors declare that they have no competing interests.

\section{Authors' contributions}

Conception and design: SDE, SL, CD. Provision of study materials or patients: GVM, BA, BH, GK, TR, KK, BF, KB, RM, HE, CT, SDE, CD, BP, MD, SL. Collection and assembly of data: SDE, GVM, CD, MKM, SL. Data analysis and interpretation: SDE, $C D, B P, S L$, Manuscript writing and final approval of manuscript: SDE, GVM, CD, $B A, B H, M K M, G K, T R, B P, K K, B F, K B, R M, M D, H E, C T, S L$.

\section{Acknowledgements}

We thank Mrs. Petra Wachs for her excellent technical assistance. This project was supported by institutional funding.

\section{Author details}

'Institute of Pathology, Charité Universitätsmedizin Berlin, Charitéplatz 1, 10117 Berlin, Germany. ${ }^{2}$ German Breast Group (GBG Forschungs GmbH), Neu-Isenburg, Germany. ${ }^{3}$ University Women's Hospital, Frankfurt am Main, Germany. ${ }^{4}$ Department of Gynecology and Obstetrics Rotkreuzklinikum München, Munich, Germany. ${ }^{5}$ Instititue of Pathology Rotkreuzklinikum München, Munich, Germany. ${ }^{6}$ Department of Gynecology and Obstetrics, Städtisches Klinikum Karlsruhe, Karlsruhe, Germany. ${ }^{7}$ Institute of Pathology, Städtisches Klinikum Karlsruhe, Karlsruhe, Germany. ${ }^{8}$ Praxisklinik Berlin, Berlin, Germany. ${ }^{9}$ Institute of Pathology, Sana Klinikum Lichtenberg, Berlin, Germany.

${ }^{10}$ Department of Gynecology and Obstetrics, University Hospital Giessen/ Marburg, Marburg, Germany. ${ }^{11}$ Insitute of Pathology, University Hospital Giessen/Marburg, Marburg, Germany. ${ }^{12}$ Department of Gynecology and Obstetrics, Universitätsklinikum Schleswig-Holstein, Kiel, Germany.

${ }^{13}$ Department of Gynecology, Universitätsklinikum Halle (Saale), Halle (Saale), Germany.

Received: 31 October 2013 Accepted: 16 July 2014

Published: 28 July 2014

\section{References}

1. Haagensen DE Jr, Mazoujian G, Holder WD Jr, Kister SJ, Wells SA Jr: Evaluation of a breast cyst fluid protein detectable in the plasma of breast carcinoma patients. Ann Surg 1977, 185:279-285.

2. Mazoujian G, Pinkus GS, Davis S, Haagensen DE Jr: Immunohistochemistry of a gross cystic disease fluid protein (GCDFP-15) of the breast. A marker of apocrine epithelium and breast carcinomas with apocrine features. Am J Pathol 1983, 110:105-112.

3. Wick MR, Lillemoe TJ, Copland GT, Swanson PE, Manivel JC, Kiang DT: Gross cystic disease fluid protein-15 as a marker for breast cancer: immunohistochemical analysis of 690 human neoplasms and comparison with alpha-lactalbumin. Hum Pathol 1989, 20:281-287.

4. Loos S, Schulz KD, Hackenberg R: Regulation of GCDFP-15 expression in human mammary cancer cells. Int J Mol Med 1999, 4:135-140.

5. Debily MA, Marhomy SE, Boulanger V, Eveno E, Mariage-Samson R, Camarca A, Auffray C, Piatier-Tonneau D, Imbeaud S: A functional and regulatory network associated with PIP expression in human breast cancer. PLoS One 2009, 4:e4696.

6. Vranic S, Schmitt F, Sapino A, Costa JL, Reddy S, Castro M, Gatalica Z: Apocrine carcinoma of the breast: a comprehensive review. Histol Histopathol 2013, 28:1393-409.

7. Honma N, Takubo K, Akiyama F, Sawabe M, Arai T, Younes M, Kasumi F, Sakamoto G: Expression of GCDFP-15 and AR decreases in larger or node-positive apocrine carcinomas of the breast. Histopathology 2005, 47:195-201.

8. Honma N, Takubo K, Arai T, Younes M, Kasumi F, Akiyama F, Sakamoto G: Comparative study of monoclonal antibody B72.3 and gross cystic disease fluid protein-15 as markers of apocrine carcinoma of the breast. APMIS 2006, 114:712-719.

9. Eusebi V, Millis RR, Cattani MG, Bussolati G, Azzopardi JG: Apocrine carcinoma of the breast. A morphologic and immunocytochemical study. Am J Pathol 1986, 123:532-541

10. Vranic S, Tawfik O, Palazzo J, Bilalovic N, Eyzaguirre E, Lee LM, Adegboyega P, Hagenkord J, Gatalica Z: EGFR and HER-2/neu expression in invasive apocrine carcinoma of the breast. Mod Pathol 2010, 23:644-653.

11. Farmer $\mathrm{P}$, Bonnefoi $\mathrm{H}$, Becette $\mathrm{V}$, Tubiana-Hulin $\mathrm{M}$, Fumoleau $\mathrm{P}$, Larsimont $\mathrm{D}$, Macgrogan G, Bergh J, Cameron D, Goldstein D, Duss S, Nicoulaz AL, Brisken C, Fiche M, Delorenzi M, Iggo R: Identification of molecular apocrine breast tumours by microarray analysis. Oncogene 2005, 24:4660-4671.

12. Doane AS, Danso M, Lal P, Donaton M, Zhang L, Hudis C, Gerald WL: An estrogen receptor-negative breast cancer subset characterized by a hormonally regulated transcriptional program and response to androgen. Oncogene 2006, 25:3994-4008.

13. Sanga S, Broom BM, Cristini V, Edgerton ME: Gene expression metaanalysis supports existence of molecular apocrine breast cancer with a role for androgen receptor and implies interactions with ErbB family. BMC Med Genomics 2009, 11:59.

14. von Minckwitz G, Blohmer JU, Raab G, Löhr A, Gerber B, Heinrich G, Eidtmann H, Kaufmann M, Hilfrich J, Jackisch C, Zuna I, Costa SD: In vivo chemosensitivity-adapted preoperative chemotherapy in patients with early-stage breast cancer: the GEPARTRIO pilot study. Ann Oncol 2005, 16:56-63. 
15. von Minckwitz G, Kümmel S, Vogel P, Hanusch C, Eidtmann $H$, Hilfrich J, Hilfrich J, Gerber B, Huober J, Costa SD, Jackisch C, Loibl S, Mehta K, Kaufmann M: Intensified neoadjuvant chemotherapy in early-responding breast cancer: phase III randomized GeparTrio study. J Natl Cancer Inst 2008, 100:552-562.

16. von Minckwitz G, Kümmel S, Vogel P, Hanusch C, Eidtmann $H$, Hilfrich J, Hilfrich J, Gerber B, Huober J, Costa SD, Jackisch C, Loibl S, Mehta K, Kaufmann M: Neoadjuvant vinorelbine-capecitabine versus docetaxeldoxorubicin-cyclophosphamide in early nonresponsive breast cancer: phase III randomized GeparTrio trial. J Natl Cancer Inst 2008, 100:542-551.

17. von Minckwitz G, Blohmer JU, Costa SD, Denkert C, Eidtmann H, Eiermann W, Gerber B, Hanusch C, Hilfrich J, Huober J, Jackisch C, Kaufmann M, Kümmel S, Paepke S, Schneeweiss A, Untch M, Zahm DM, Mehta K, Loibl S: Response-Guided Neoadjuvant Chemotherapy for Breast Cancer. J Clin Oncol 2013, 31:3623-3630.

18. Wolff AC, Hammond ME, Schwartz JN, Hagerty KL, Allred DC, Cote RJ, Dowsett M, Fitzgibbons PL, Hanna WM, Langer A, McShane LM, Paik S, Pegram MD, Perez EA, Press MF, Rhodes A, Sturgeon C, Taube SE, Tubbs R, Vance GH, van de Vijver M, Wheeler TM, Hayes DF: American Society of Clinical Oncology; College of American Pathologists. American Society of Clinical Oncology/College of American Pathologists guideline recommendations for human epidermal growth factor receptor 2 testing in breast cancer. J Clin Oncol 2007, 25:118-145.

19. Hammond ME, Hayes DF, Dowsett M, Allred DC, Hagerty KL, Badve S, Fitzgibbons PL, Francis G, Goldstein NS, Hayes M, Hicks DG, Lester S, Love R, Mangu PB, McShane L, Miller K, Osborne CK, Paik S, Perlmutter J, Rhodes A, Sasano H, Schwartz JN, Sweep FC, Taube S, Torlakovic EE, Valenstein P, Viale G, Visscher D, Wheeler T, Williams RB, Wittliff JL, Wolff AC: American Society of Clinical Oncology/College Of American Pathologists guideline recommendations for immunohistochemical testing of estrogen and progesterone receptors in breast cancer. J Clin Oncol 2010, 28:2784-2795.

20. Loibl S, Müller BM, von Minckwitz G, Schwabe M, Roller M, Darb-Esfahani S, Ataseven B, du Bois A, Fissler-Eckhoff A, Gerber B, Kulmer U, Alles JU, Mehta $K$, Denkert C: Androgen receptor expression in primary breast cancer and its predictive and prognostic value in patients treated with neoadjuvant chemotherapy. Breast Cancer Res Treat 2011, 130:477-487.

21. Huo L, Zhang J, Gilcrease MZ, Gong Y, Wu Y, Zhang H, Resetkova E, Hunt KK, Deavers MT: Gross cystic disease fluid protein-15 and mammaglobin A expression determined by immunohistochemistry is of limited utility in triple-negative breast cancer. Histopathology 2013, 62:267-274.

22. Lewis GH, Subhawong AP, Nassar $H$, Vang R, Illei PB, Park BH, Argani P: Relationship between molecular subtype of invasive breast carcinoma and expression of gross cystic disease fluid protein 15 and mammaglobin. Am J Clin Pathol 2011, 135:587-591.

23. Weigelt B, Horlings HM, Kreike B, Hayes MM, Hauptmann M, Wessels LF, de Jong D, Van de Vijver MJ, Van't Veer LJ, Peterse JL: Refinement of breast cancer classification by molecular characterization of histological special types. J Pathol 2008, 216:141-150.

24. Pagani A, Sapino A, Eusebi V, Bergnolo P, Bussolati G: PIP/GCDFP-15 gene expression and apocrine differentiation in carcinomas of the breast. Virchows Arch 1994, 425:459-465.

25. Fritzsche FR, Thomas A, Winzer KJ, Beyer B, Dankof A, Bellach J, Dahl E, Dietel M, Kristiansen G: Co-expression and prognostic value of gross cystic disease fluid protein 15 and mammaglobin in primary breast cancer. Histol Histopathol 2007, 22:1221-1230.

26. Luo MH, Huang YH, Ni YB, Tsang JY, Chan SK, Shao MM, Tse GM: Expression of mammaglobin and gross cystic disease fluid protein-15 in breast carcinomas. Hum Pathol 2013, 44:1241-1250.

27. Hall RE, Clements JA, Birrell SN, Tilley WD: Prostate-specific antigen and gross cystic disease fluid protein-15 are co-expressed in androgen receptor-positive breast tumours. Br J Cancer 1998, 78:360-365.

28. Lakhani S, Ellis IO, Tan PH, van de Vijver MJ: WHO Classification of Tumors of the Breast. Lyon: IARC Press; 2012.

\section{doi:10.1186/1471-2407-14-546}

Cite this article as: Darb-Esfahani et al:: Gross cystic disease fluid protein 15 (GCDFP-15) expression in breast cancer subtypes. BMC Cancer 2014 14:546.

\section{Submit your next manuscript to BioMed Central and take full advantage of:}

- Convenient online submission

- Thorough peer review

- No space constraints or color figure charges

- Immediate publication on acceptance

- Inclusion in PubMed, CAS, Scopus and Google Scholar

- Research which is freely available for redistribution 